\title{
A Influência da Traqueostomia Precoce no Desmame Ventilatório de Pacientes com Traumatismo Craniencefálico Grave*
}

\author{
The Influence of Early Tracheostomy in the Weaning \\ of Patients with Severe Traumatic Brain Injury
}

Renata Lenize Pasini', Yvens Barbosa Fernandes², Sebastião Araújo ${ }^{3}$, Silvia Maria de Toledo Piza Soares ${ }^{4}$

\section{RESUMO}

JUSTIFICATIVA E OBJETIVOS: Atualmente, ainda é questionável se a traqueostomia precoce (TP) pode influenciar no desmame ventilatório ou no tempo de internação hospitalar de pacientes com traumatismo cranioencefálico (TCE). O objetivo primário deste estudo foi verificar se a TP influencia o tempo de ventilação mecânica (VM) em pacientes com TCE grave.

MÉTODO: Estudo prospectivo, observacional, incluindo 33 pacientes com pontuação admissional na escala de coma de Glasgow (ECG) $\leq 10$, analisados segundo o momento de realização traqueostomia, após a intubação traqueal (precoce: $\mathrm{t} \leq 6$ dias; intermediária: $\mathrm{t}=7$ a 11 dias; tardia: $\mathrm{t} \geq 12$ dias) e o desmame ventilatório.

RESULTADOS: O tempo total de VM foi menor no

1. Fisioterapeuta Especialista em Fisioterapia Respiratória em Terapia Intensiva Adulto pela FCM-UNICAMP; Mestranda em Ciências Médicas pela FCM-UNICAMP.

2. Doutor em Neurologia e Assistente em Neurocirurgia do HC-UNICAMP.

3. Professor Assistente Doutor do Departamento de Cirurgia da FCM-UNICAMP e Assistente da UTI-Adulto do HC-UNICAMP.

4. Mestre, Fisioterapeuta Especialista em Fisioterapia Respiratória em Terapia Intensiva Adulto do HC-UNICAMP.

*Recebido da Unidade de Terapia Intensiva do Hospital de Clínicas da Universidade Estadual de Campinas (HC-UNICAMP), Campinas, SP

Apresentado em 02 de abril de 2007

Aceito para publicação em 23 de maio de 2007

Endereço para correspondência:

Dra. Renata Lenize Pasini

Rua Iguape $\mathrm{n}^{\circ} 3 \mathrm{~A} 4$, Jardim Paulista

13090-090 Ribeirão Preto, SP

E-mail: renata.lenize@terra.com.br

(C)Associação de Medicina Intensiva Brasileira, 2007 grupo TP ( $n=10 ; p<0,0001)$. No grupo TP, a menor pontuação na ECG (média de 5,3 $\pm 2,5$ ) esteve negativamente correlacionada com o tempo de internação hospitalar $(p=0,02)$.

CONCLUSÕES: A traqueostomia precoce pode reduzir os tempos de ventilação mecânica, mas não influencia o tempo de internação hospitalar em pacientes com traumatismo cranioencefálico grave.

Unitermos: desmame, traqueostomia, traumatismo cranioencefálico, ventilação mecânica.

\section{SUMMARY}

BACKGROUND AND OBJECTIVES: Actually, It's doubtful if early tracheostomy (ET) can influence mechanical ventilation (MV) weaning time or the hospital length of stay in patients with traumatic brain injury (TBI). The main objective of this trial was to verify the influence of ET on weaning time of patients with severe TBI.

METHODS: Prospective, observational study, including 33 patients with severe TBI (GCS $\leq 10$ at hospital admission), evaluated according to tracheostomy performance time (early: $\mathrm{t} \leq 6$ days; intermediate: $\mathrm{t}=$ 7 to 11 days; and late: $t \geq 12$ days) and the weaning from MV.

RESULTS: Total ventilation mechanical time has been reduced in the early tracheostomy group $(n=10 ; p<$ 0.0001 ). A lower GCS punctuation (mean $5.3 \pm 2.5$ ) in ET group has been negatively correlated with hospital length of stay (LOS) $(p=0.02)$.

CONCLUSIONS: Early tracheostomy can decrease mechanical ventilation time, but does not influence hospital LOS in patients with severe traumatic brain injury.

Key Words: mechanical ventilation, tracheostomy, traumatic brain injury, weaning. 


\section{INTRODUÇÃO}

A ventilação e a oxigenação encefálica em neuroemergência são prioridades no atendimento de pacientes com traumatismo cranioencefálico (TCE), ou qualquer outro tipo de lesão traumática grave, com a finalidade de se diminuir o sofrimento encefálico e as suas complicações secundárias ${ }^{1}$.

Para a manutenção de boa condição ventilatória na fase aguda do trauma, o suporte ventilatório artificial é necessário, já que a capacidade de manter a permeabilidade das vias aéreas e a troca gasosa é deficiente nesse momento.

Considerando que o manuseio desses pacientes é delicado, exigindo monitorização rigorosa e possibilidade de intervenção cirúrgica, eles acabam permanecendo por longos períodos em ventilação mecânica (VM). Nesses casos, a traqueostomia é freqüentemente indicada, pois facilita a remoção de secreções pulmonares, diminui o desconforto com a via aérea artificial e aumenta a mobilidade do paciente no leito. Esses benefícios possibilitam a redução do tempo de ventilação artificial, da incidência de pneumonia e do tempo de internação hospitalar ${ }^{2}$. No entanto, embora comumente realizada, não há consenso na literatura quanto aos benefícios da traqueostomia nos pacientes com TCE.

Dessa forma, o objetivo primário da presente investigação foi verificar a influência da traqueostomia realizada precocemente no tempo de desmame da VM em pacientes com TCE grave internados em unidade de terapia intensiva (UTI) geral; e, secundariamente, sua influência no tempo de internação hospitalar.

\section{MÉTODO}

Trata-se de um estudo prospectivo, observacional, não intervencionista, realizado na Unidade de Terapia Intensiva do Hospital de Clínicas da UNICAMP. O protocolo de pesquisa foi aprovado pelo Comitê Institucional de Ética em Pesquisa, sob parecer $n^{\circ}$ 094/2004. Foram incluídos no estudo pacientes vítimas de TCE, admitidos na UTI no período de junho de 2005 a agosto de 2006, com pontuação na escala de coma de Glasgow (ECG) $\leq 10$ no momento da admissão hospitalar, com idade superior a 14 anos e submetidos à VM por mais de 48 horas (Figura 1).

Foram excluídos do estudo os pacientes extubados durante o período de observação, aqueles que evoluíram para morte encefálica, os que por alguma razão foram traqueostomizados no momento da admissão ou aqueles em que não foi possível a obtenção do termo de consentimento livre e esclarecido.

A traqueostomia foi realizada no centro cirúrgico utilizando-se a técnica cirúrgica convencional. Após a sua realização, os pacientes foram acompanhados diariamente por pesquisador, com a finalidade de observar o momento da retirada da VM, estipulado como aquele em que o paciente foi colocado em nebulização por meio de tubo-T.

Também foram anotados os tempos totais de ventilação mecânica, a incidência de infecção pulmonar durante a permanência na UTI e o tempo de internação hospitalar.

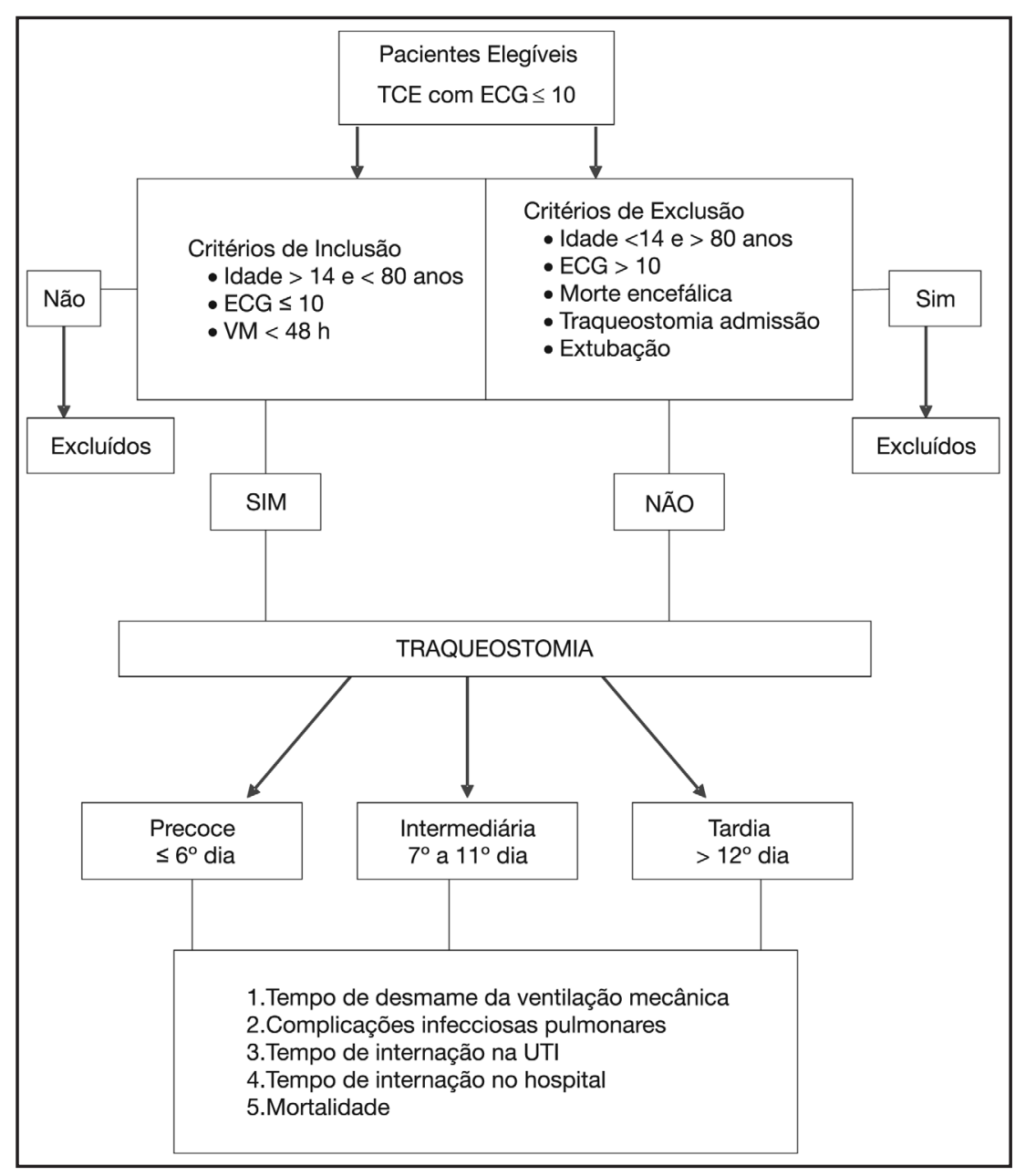

Figura 1 - Fluxograma do Estudo 
Os critérios para avaliação da incidência de complicações infecciosas associadas à VM incluíram o uso, tipos de antibióticos, variabilidade de doses e tempos de utilização pelos pacientes durante a permanência na UTI, assim como as alterações no leucograma e radiografia de tórax e a presença do diagnóstico de pneumonia anotado no prontuário do paciente pela equipe médica da UTI.

Para a análise dos resultados, os pacientes foram agrupados de acordo com o tempo entre a intubação traqueal e a realização da traqueostomia denominada precoce (TP), intermediária (TI) e tardia (TT). A TP foi aquela realizada até $\circ 6^{\circ}$ dia de intubação traqueal, a TI do $7^{\circ}$ ao $11^{\circ} \mathrm{dia}$, e a $T$ após o $12^{\circ}$ dia de intubação.

Para a análise estatística foi utilizado o teste Exato de Fisher para variáveis categóricas, Análise de Variância (ANOVA) com transformação em postos (ranks) para variáveis contínuas, e o teste Tukey nos ranks para identificar os grupos diferentes.

$\mathrm{Na}$ comparação de amostras pareadas foi utilizado o teste não paramétrico de Wilcoxon. Foi também realizado o coeficiente de correlação de Spearman (associação linear) entre variáveis ECG e APACHE II. Tal coeficiente assume valores $-1 \mathrm{a}+1$, sendo que quanto mais próximos de zero, menos correlacionadas são as variáveis, e quanto mais próximo de +1 ou -1 , mais correlacionadas são as variáveis.

O nível significativo adotado para os testes estatísticos foi de $5 \%$, ou seja, $p \leq 0,05$.

\section{RESULTADOS}

Durante o período de observação, 72 pacientes com TCE preencheram os critérios de inclusão no estudo, porém 39 foram excluídos pelos seguintes motivos: não realização de traqueostomia $(n=25)$ e evolução para morte encefálica $(n=14)$. Ao final, foram analisados 33 pacientes, divididos em três grupos: TP $(n=$ 10), TI $(n=12)$ e TT $(n=11)$.

Os três grupos foram comparáveis quanto às seguintes variáveis: idade, sexo, pontuação na ECG e APACHE II (Tabela 1).

No grupo TP observou-se uma correlação negativa entre a pontuação na ECG inicial $(5,3 \pm 2,5)$ e o tempo de internação hospitalar ( $p=0,027$ ). No grupo submetido à $\mathrm{TI}$, menor tempo de internação hospitalar esteve associado a pacientes mais jovens $(p=0,0016)$. Nos pacientes submetidos à TT não foi observada correlação significativa com o tempo de internação hospitalar (Tabela 2).

Tabela 1 - Dados Demográficos, Índice APAHE II e Pontuação na ECG dos Pacientes Estudados ( $n=33)$.

\begin{tabular}{|c|c|c|c|c|}
\hline Variáveis & TP & $\mathrm{TI}$ & Tा & $\mathrm{p}$-valor \\
\hline Número de pacientes & 10 & 12 & 11 & \\
\hline Idade & $30,7 \pm 14$ & $39 \pm 18,4$ & $37,7 \pm 18,4$ & $0,51^{*}$ \\
\hline Sexo & & & & $1,0^{\star \star}$ \\
\hline Feminino & 1 & 2 & 2 & \\
\hline Masculino & 9 & 10 & 9 & \\
\hline APACHE II & $20,4 \pm 4,2$ & $20,8 \pm 4,4$ & $20,9 \pm 3,6$ & $0,95^{\star}$ \\
\hline ECG & $5,3 \pm 2,5$ & $6,8 \pm 2,5$ & $8,3 \pm 2,3$ & $0,04^{*}$ \\
\hline
\end{tabular}

$\mathrm{TP}=$ traqueostomia precoce; $\mathrm{TI}=$ traqueostomia intermediaria; $\mathrm{TT}$ = traqueostomia tardia

${ }^{*}$ Kruskal-Wallis, ${ }^{* \star}$ Exato de Fisher

$\mathrm{APACHE}=$ Acute Physiology and Chronic Health Evaluation; ECG = escala de coma de Glasgow

Tabela 2 - Resultado da Correlação de Spearman (r/p-valor) para o Tempo de Internação Hospitalar, Intubação e Desmame Ventilatório Relacionados à Traqueostomia Precoce e à Traqueostomia Intermediária.

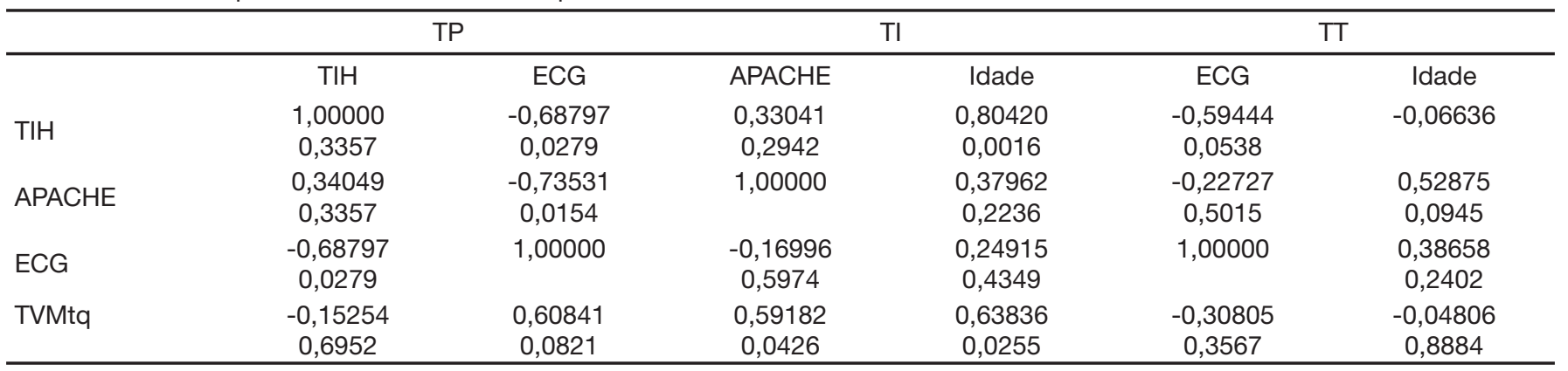

$\mathrm{TP}=$ traqueostomia precoce; $\mathrm{TI}=$ traqueostomia intermediaria; $\mathrm{TT}=$ traqueostomia tardia; $\mathrm{TIH}=$ tempo de internação hospitalar; $\mathrm{APACHE}=$ Acute Physiology and Chronic Health Evaluation; ECG = escala de coma de Glasgow; TVMtq = tempo de desmame ventilatório da traqueostomia. 
O tempo de internação hospitalar mostrou uma tendência a ser menor para o grupo da TP, com média de $34,2 \pm 23,4$ dias, porém sem diferença estatisticamente significativa (Tabela 3).

Tabela 3 - Resultado da Análise de Variância, Testes Exato de Fisher e Tukey para os Tempos Ventilatórios e Tempo de Internação Hospitalar nos Grupos Estudados (dias).

\begin{tabular}{lcccc}
\hline & TP & TI & TT & p-valor \\
\hline $\begin{array}{l}\text { Número de } \\
\text { pacientes }\end{array}$ & 10 & 12 & 11 & \\
TTO & $4,3 \pm 1,2$ & $8,7 \pm 1,4$ & $14,5 \pm 2,7$ & - \\
TVMtq & $4,2 \pm 2,0$ & $5,5 \pm 4,9$ & $6,5 \pm 2,9$ & 0,2277 \\
TVMtotal & $8,4 \pm 2,4$ & $14,2 \pm 5,4$ & $21,0 \pm 3,3$ & $<0,0001$ \\
TIH & $34,2 \pm 23,4$ & $58,2 \pm 60,9$ & $60,6 \pm 26,6$ & 0,0749 \\
\hline
\end{tabular}

$\mathrm{TP}=$ traqueostomia precoce; $\mathrm{TI}=$ traqueostomia intermediaria; $\mathrm{TT}=$ traqueos tomia tardia; TTO = tempo de tubo traqueal; TVMtotal = tempo de ventilação mecânica total; TVMtq = tempo de desmame ventilatório da traqueostomia; $\mathrm{TIH}=$ tempo de internação hospitalar.

O tempo total de VM (com o tubo traqueal e traqueostomia) foi menor no grupo submetido à TP $(p<0,0001)$ em relação aos demais grupos. Entretanto, quanto ao tempo de desmame da VM após a realização da traqueostomia não se observou diferença significativa entre os grupos (Tabela 3).

Quanto a presença de infecção pulmonar, 19 pacientes tiveram o diagnóstico de pneumonia anotado em prontuário, sendo quatro pacientes no grupo de TP, oito no grupo de TI e sete no grupo de TT. Não houve diferença estatisticamente significativa entre a presença de pneumonia e o momento da realização da traqueostomia ( $p$-valor = 0,4554; Exato de Fisher).

Dos 33 pacientes, 30 tiveram alta hospitalar, enquanto três faleceram, sendo que um deles faleceu antes do desmame ventilatório no grupo TP. Não houve diferença estatisticamente significativa associada entre o tempo de traqueostomia e mortalidade (Tabela 4).

Tabela 4 - Tipo de Traqueostomia Versus Sobrevida.

\begin{tabular}{lcccc}
\hline Óbito & TP $(n=10)$ & TI $(n=12)$ & TT $(n=11)$ & Total \\
Não & $9(90 \%)$ & $11(92 \%)$ & $10(91 \%)$ & $30(91 \%)$ \\
Sim & $1(10 \%)$ & $1(8 \%)$ & $1(9 \%)$ & $3(9 \%)$ \\
Total & $10(30 \%)$ & $12(36 \%)$ & $11(33 \%)$ & $33(100 \%)$ \\
\hline
\end{tabular}

$\mathrm{TP}=$ traqueostomia precoce; $\mathrm{Tl}=$ traqueostomia intermediaria; $\mathrm{TT}=$ traque ostomia tardia.

Teste Exato de Fisher $\mathrm{p}$-valor $=1,0000$.

\section{DISCUSSÃO}

A traqueostomia é um procedimento comumente utilizado em pacientes internados em UTI, principalmente em pacientes com lesão encefálica grave, uma vez que eles necessitam tempos prolongados de VM. No entanto, embora seja freqüentemente recomendada, existem controvérsias sobre as verdadeiras vantagens e desvantagens desse procedimento no tratamento de pacientes com TCE, assim como não há consenso sobre a terminologia empregada para definir o tempo de realização da traqueostomia.

A traqueostomia precoce é classificada como sendo aquela realizada até o período máximo de sete a 10 dias $^{3-6}$, quatro dias ${ }^{7,8}$ ou três dias ${ }^{9,10}$ de VM. No presente estudo, classificou-se como traqueostomia precoce o procedimento realizado até sete dias de VM. Já a traqueostomia tardia vem sendo referida como aquela realizada no período de sete ${ }^{8,9}, 10$ ou 13 e até 28 dias $^{2,11}$ após a intubação traqueal e o início da VM. Nesta pesquisa, a TT foi definida como aquela ocorrendo a partir do $12^{\circ}$ dia de VM.

Entretanto, com essa terminologia, é plausível uma interpretação errônea dos dados, pois o que é considerado um procedimento precoce em alguns estudos pode ser tardia para outros. Dessa forma, determinou-se um período intermediário (sete a 11 dias de VM) para classificar a traqueostomia realizada no período de transição entre a precoce e a tardia.

Estudos acreditam que a realização mais precoce do procedimento pode prevenir complicações decorrentes da intubação traqueal, tais como: ulceração da mucosa, edema de laringe, disfunção da mecânica respiratória e, em menor escala, a ocorrência de estridor laríngeo pós-extubação ${ }^{12}$. Segundo Ross e col. ${ }^{13}$ o fator determinante das complicações é o trauma provocado pelo tubo traqueal, sendo este ocasionado pelo movimento e pressão do tubo na mucosa, podendo gerar necrose tecidual e estenose subglótica e laríngea.

Num estudo sistemático, concluiu-se que não há evidências suficientes para embasar o pressuposto de que o tempo da traqueostomia afeta a duração da VM ou a extensão da lesão na via aérea em pacientes graves ${ }^{6}$.

Quanto ao tempo de internação hospitalar, os pacientes submetidos à TP mostraram uma tendência de menor tempo em comparação com os demais grupos, porém sem diferença estatisticamente significativa entre eles. Esses dados são compatíveis com aqueles da literatura ${ }^{3,14-16}$. No entanto, esta redução foi somente observada em pacientes que apresentaram maiores pontuações na ECG. Este fato é aceitável, visto que quanto mais acentuado o grau de comprometimento neurológico (menor pontuação na 
ECG de admissão), maior será o tempo necessário para a recuperação desse estado.

Outra variável significativa encontrada no estudo foi a relação do tempo de internação com a idade, onde foi observado maior tempo de internação para pacientes mais velhos no grupo da TI. Isto pode ser explicado, pois neste grupo o intervalo de idade (17 a 78 anos) abrange o intervalo de idade do grupo precoce (17 a 60 anos) e do tardio (18 a 78 anos) e, em média, a idade do grupo TI foi maior, apesar de não apresentar diferença estatística significativa.

Em oposição, o estudo prévio de Frutos-Vivar e col. ${ }^{17}$ incluindo pacientes adultos internados em UTI de 20 países da Europa, relatou que pacientes com traqueostomia tiveram maior tempo de internação, tanto na UTI quanto no hospital, quando comparados com pacientes sem traqueostomia ${ }^{8,18-20}$. Outros estudos relataram que a traqueostomia não interfere no tempo de internação hospitalar ${ }^{5,21}$.

Vários estudos retrospectivos parecem indicar que a traqueostomia diminui o tempo de $\mathrm{VM}^{4,814-16,22}$. No presente estudo, a média de tempo total do uso de VM foi menor no grupo TP em relação aos outros grupos, porém sem diferenças estatísticas significativas quanto aos tempos de desmame pós-traqueostomia, contrastando com a literatura.

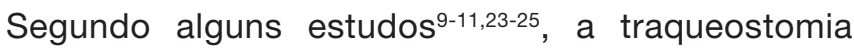
influencia na incidência de infecção respiratória e, desse modo, estudos vêm enfatizando a proposta da sua realização mais precoce, com a finalidade de reduzir a ocorrência de pneumonias associadas à VM, pois se acredita que tal benefício ocorre devido ao fato da cânula da traqueostomia facilitar o toalete brônquico espontâneo e a remoção de secreções traqueais, além de diminuir o risco de bronco-aspirações de forma repetida.

No presente estudo, a incidência de pneumonia não foi significativamente diferente entre os grupos de traqueostomia, embora esta tenha sido menor no grupo TP.

Möller e col. ${ }^{15}$ avaliaram, dentre outros parâmetros, a incidência de pneumonia associada à VM em pacientes submetidos à traqueostomia precoce versus traqueostomia tardia, e verificaram que a incidência de pneumonia esteve mais associada ao procedimento tardio. No entanto, relataram que esta diferença significativa estatisticamente, foi encontrada apenas no subgrupo de pacientes traqueostomizados nas primeiras $48 \mathrm{~h}$ de VM e que não houve decréscimo na incidência de pneumonia nos pacientes traqueosto- mizados entre três a sete dias de VM.

Em outro estudo, foi avaliada a ocorrência de pneumonia nosocomial, assim como as complicações do tempo prolongado de VM em pacientes com trauma de múltiplos órgãos, os autores concluíram que esta complicação foi menor no grupo de traqueostomia precoce (realizada até o quarto dia de VM) (19\%) em relação à traqueostomia tardia $(59 \%)^{8}$.

Alguns autores que também avaliaram a incidência de pneumonia relacionada à VM defendem a hipótese de que a traqueostomia não interfere na incidência da infecção em pacientes com suporte ventilatório mecânico ${ }^{3,21,22}$.

De acordo com a literatura, o índice de mortalidade dos pacientes submetidos à traqueostomia precoce é baixo, quando comparado aos pacientes não traqueostomizados ou aqueles submetidos ao procedimento mais tardiamente, com este índice sendo analisado tanto durante o procedimento quanto após a sua realização ${ }^{3,16}$. Segundo Arabi e col. ${ }^{4}$ a baixa mortalidade dos pacientes submetidos à traqueostomia está relacionada ao seu critério de seleção, ou seja, os candidatos ao procedimento provavelmente tinham maiores probabilidades de sobrevivência. No presente estudo, a taxa de sobrevida dos pacientes com TCE submetidos à traqueostomia foi alta, não havendo diferença significativa entre os grupos.

O presente trabalho apresentou importantes limitações, dentre as quais podem ser destacadas: 1) tratou-se de um estudo de observação, sem grupocontrole ou aleatorização; 2) a casuística foi relativamente pequena, não permitindo adequada homogeneização dos grupos, como, por exemplo, uma menor pontuação inicial na ECG constatada nos pacientes submetidos à TP, indicando que houve maior tendência a realizá-la naqueles pacientes mais graves, o que certamente enviesa a interpretação dos resultados finais; e 3) o diagnóstico de pneumonia foi presumido a partir de dados clínicos, sem comprovação bacteriológica.

Diante do exposto, levando-se em conta as limitações do presente trabalho, os resultados mostraram que a TP pode reduzir os tempos de VM, mas não o de internação hospitalar em pacientes com TCE grave com menores pontuações na ECG. Novos estudos prospectivos, controlados e aleatórios, com protocolos de investigação bem delineados e incluindo maior número de pacientes são de fundamental importância para o esclarecimento desta questão. 


\section{A INFLUÊNCIA DA TRAQUEOSTOMIA PRECOCE NO DESMAME VENTILATÓRIO DE PACIENTES COM TRAUMATISMO CRANIENCEFÁLICO GRAVE}

\section{REFERÊNCIAS}

01. Capone Neto A, Knobel E - Eventos Hemodinâmicos Sistêmicos em Neurointensivismo, em: Cruz J - Neurointensivismo. CBMI Série Clínicas Brasileiras de Medicina Intensiva, São Paulo: Atheneu, 2002;49-61.

02. Heffner JE - The role of tracheotomy in weaning. Chest, 2001;120:(Suppl6):477S-481S.

03. Gurkin SA, Parikshak M, Kralovich KA et al - Indicators for tracheostomy in patients with traumatic brain injury. Am Surg, 2002;68:324-329.

04. Arabi Y, Haddad S, Shirawi N et al - Early tracheostomy in intensive care trauma patients improves resource utilization: a cohort study and literature review. Crit Care, 2004;8: R347-R352.

05. Barquist ES, Amortegui J, Hallal A et al - Tracheostomy in ventilator dependent trauma patients: a prospective, randomized intention-to-treat study. J Trauma, 2006;60:91-97.

06. Maziak DE, Meade MO, Todd TR - The timing of tracheostomy: a systematic review. Chest, 1998;114:605-609.

07. Heffner JE - Medical indications for tracheostomy. Chest, 1989;96:186189.

08. Lesnik I, Rappaport W, Fulginiti J et al - The role of early tracheostomy in blunt, multiple organ trauma. Am Surg, 1992;58:346-349.

09. Kluger $\mathrm{Y}$, Paul DB, Lucke $\mathrm{J}$ et al - Early tracheostomy in trauma patients. Eur J Emerg Med, 1996;3:95-101.

10. Rodriguez JL, Steinberg SM, Luchetti LA et al - Early tracheostomy for primary airway management in the surgical critical care setting. Surgery, 1990;108:655-659.

11. Dunham CM, Ransom KJ - Assessment of early tracheostomy in trauma patients: a systematic review and meta-analysis. Am Surgeon, 2006;72:276-281.

12. Kriner EJ, Shafazand S, Colice GL - The endotracheal tube cuff-leak test as a predictor for postextubation stridor. Respir Care, 2005;50:16321638.
13. Ross BJ, Barker DE, Russell WL et al - Predicting of long-term ventilatory support in trauma patients. Am Surg, 1996;62:19-25.

14. Brook AD, Sherman $\mathrm{G}$, Malen $\mathrm{J}$ et al - Early versus late tracheostomy in patients who require prolonged mechanical ventilation. Am J Crit Care, 2000;9:352-359.

15. Moller MG, Slaikeu JD, Bonelli P et al - Early tracheostomy versus late tracheostomy in the surgical intensive care unit. Am J Surg, 2005;189:293-296.

16. Flaatten $\mathrm{H}$, Gjerde $\mathrm{S}$, Heimdal $\mathrm{JH}$ et al - The effect of tracheostomy on outcome in intensive care unit patients. Acta Anaesthesiol Scand, 2006;50:92-98.

17. Frutos-Vivar F, Esteban A, Apezteguia C et al - Outcome of mechanically ventilated patients who require a tracheostomy. Crit Care Med, 2005;33:290-298.

18. LoCicero J, McCann B, Massad M et al - Prolonged ventilatory support after open-heart surgery. Crit Care Med, 1992;20:990-992.

19. Kollef MH, Ahrens TS, Shannon W - Clinical predictor and outcomes for patients requiring tracheostomy in the intensive care unit. Crit Care Med, 1999;27:1714-1720.

20. Engoren M, Arslanian-Engoren C, Fenn-Buderer N - Hospital and long-term outcome after tracheostomy for respiratory failure. Chest, 2004;125:220-227.

21. Sugerman HJ, Wolfe L, Pasquale MD et al - Multicenter, randomized, prospective trial of early tracheostomy. J Trauma, 1997;43:741-747.

22. Bouderka MA, Fakhir B, Bouaggad A et al - Early tracheostomy versus prolonged endotracheal intubation in severe head injury. J Trauma, 2004;57:251-254.

23. Armstrong PA, McCarthy MC, Peoples JB - Reduced use of resources by early tracheostomy in ventilator-dependent patients with blunt injury. Surgery, 1998;124:763-767.

24. Teoh WH, Goh KY, Chan C - The role of early tracheostomy in critically ill neurosurgical patients. Ann Acad Med Singapore, 2001;30:234-238.

25. Pierson DJ - Tracheostomy and weaning. Respir Care, 2005;50:526533. 